\title{
Investigating the Linguistics Features of Bayza Dialect
}

\author{
Ghorbanali Ebrahimi \\ Persian Literature Department, Najafabad Branch, Islamic Azad University, Najafabad, Iran \\ Email: tabatabaeiomid@yahoo.com
}

\begin{abstract}
Bayza is a dialect spoken in the southwest of Iran in Fars province. This paper examines phonetic peculiarities, morphological system and vocabulary of this dialect. The dialect of Bayza has not yet been studied and described by either Iranians or non-Iranians researchers. Bayza is a region in Fars province and is located 50 kilometers northwest of Shiraz. This region is considered as one of the most ancient centers of Iran. Its Elamite-Achaemenian name is Anzan or Anshan. During Sassanid period, it was called "Darespid" and later on it was changed to Bayza by Arabs. Bayza is a fertile region with a mild and pleasant climate. Its population is around 45000 , and the main job of its people is farming and animal husbandry. The Bayza dialect is among the dialects associated with the southwest Persian languages. In this paper, Bayza dialect is studied at the level of phonemics, phonetics, morphology and vocabulary.
\end{abstract}

Index Terms - west Persian languages, southwest Persian languages, Bayzai, phonetic system, morphology, vocabulary

\section{INTRODUCTION AND BACKGROUND}

Bayza is located in the northwest of Shiraz, Iran. In terms of country divisions, it is under the control of Sepidan Township. It is about 60/000 hectares in area and between 1220 to 1580 meters above the sea level. The Bayza plain is surrounded by mountains from three directions: the west, the north and the south. It has semi-dry climate, long summers and cold winters. Bayza plain has no constantly-running river and is irrigated by Kanats and deep wells. Its population is about 45000. The main job of its people is farming and animal husbandry. Bayza is considered as one of the most important centers of agriculture in Fars province in Iran.

The name "Bayza" belongs to the Islamic era and it has, for the first time, been mentioned in the early centuries of the advent of Islam. The first source which has cited the name of Bayza is "Tarikh-e-Tabari" in the fourth century A.H (Tabari, 1983 p. 581). Ibn-e-Balkhi's Farsnameh and Hamdollah-e-Mostofi's Nozhat -al-Gholub have argued that the name of Bayza has been chosen because of its white soil. The former name of Bayza, as quoted by Ibn-e-Hughal, was "Daresfid" (Ibn-e-Hughal, 1987), which according to Kalman Hovar, was translated "Bayza" by the Arabs (Hovar, Kalman , 1996: p.141).

Harrold Bailey has also written a detailed article in this regard and translated "Daresfid" as "wite Palace" (Bailey, Harrold, 2002: pp. 644-756).

Before Darisfid, Yaghut expressed the idea that the name of Bayza was Nisa, which means "the place of rest, or camp" (Bailey, Harrold, 2005: pp.644-756). On the basis of archeslogical excavations carried out by William Sumner in 1968-1978, it was made clear that "Malian hill" in Bayza region is the very "Anshan or Arzan", one of the two capitals of Elamiate (Sumner, 1988: a) and (Reiner, 1973) by the occupation of which Achemenian could stabilize their position in Fars.

The current dialect in Bayza is Bayzai, but people also speak in Lori as well as Lori-Bayzai, in the west northwest regions and Turkish and Turkish-Bayzai in some villages of the eastern regions or accents.

Although Bayza has not so far been listed as a Persian dialect regarding the phonetic changes of this dialect, we should consider it as one of the local dialects of Fars province which itself is a branch of southwest Persian dialects. Accordingly, this dialect is a relative of Sarvestani, Zarghani, Sabunati, Fasai, Jahromi, Neyrizi and Bavanati dialects. This classification is on the basis of a combination of family- language relations, geographical neighboring, ethnic identity and phonetic and vocabulary system.

The collection of southwest Persian dialects is within the larger collection of western Persian dialects.

The data of this research was collected in Bayza region during a few years. (2001-2003)

\section{RESEARCH QUESTIONS}

1. What are the differences between Bayzai dialect and the standard Persian dialect?

2. Do the description and analysis of Bayzai dialect contribute to the description and recognition of unknown aspects of standard Persian Language?

\section{A. Phonetic Changes}


1.1 vowels

The Bayzai dialect, like Standard Farsi, has 3 short vowels o, e, a, and 3 long vowels i, u and a. It has also 4 diphthongs ey, ow, āy and oy. The mentioned cases can be considered as diphthongs only from the phonetic, but not phonemic aspect because actually each of them consists of one simple vowel and one of the semi-vowels "w, y".

Close

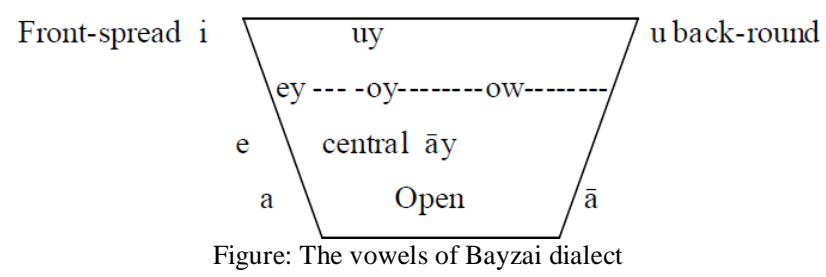

1.2. phonemic description of vowels

1.2.1. /a/ front, low, spread, open

1. /a/-/o/ : sar /sær/ [head] - sor / o / [slippery]

2. /a/-/ā/ : xar /khær/ [donkey] - xār /khar/ [thorn]

3. /a/-/e/ : kar /kær/ [deaf] - ker /ker/ [hidden, corner]

1.2.2. $/ \overline{\mathrm{a}} /$ : back, low, spread, open

1. /ā/-/a/ : borrā /borra/ [winner] - borra /borro/ [harvester]

2. /ā/-/e/ : xār /khar/ [thorn] - xer /kher/ [throat]

3. /ā/-/u/ : zār /zar/ [deplorable (sad)] - zur /zur/ [force]

1.2.3. /e/ : front, central, spread

1. /e/-/i/ : lem /lem/ [manner] - $\lim / \lim /$ [harmful]

2. /e/-/i/ : ker /ker/ [hidden] - kor /kor/ [child]

3. /e/-/u/ : del /del/ [heart] - dul /dul/ [leather bucket]

1.2.4. /i/ : Front, high, close, spread

1. /i/-/e/ : pir /pīr/ [old] - per /per/ [ears of wheat]

2. $/ \mathrm{i} /-/ \mathrm{o} /: \operatorname{dim} / \mathrm{dīm} /$ [edge] - dom /dom/ [tail]

3. /i/-/o/ : šir / /īr/ [milk] - šur / fur/ [salty]

1.2.5. /o/ : back, round, half -open

1. /o/-/u/ : šor / for/ [flow of water] - šur / fur/ [salty]

2. /o/-/a/ : pol /pol/ [bridge] - pal /pæl/ [braid]

3 ./o/-/e/ : kot /kot/ [root] - ket /ket/ [edge]

1.2.6. $/ \mathrm{u} /$ : Back, high, round, close

1. /u/-/o/ : buna /bunə/ [pretext] - bona /bonə/ [temporary place of living]

2. /u/-/ā/ : sul /sul/ [information] - sāl /s $\Lambda \mathrm{l} /$ [year]

3. /u/-/e/ : ruz /ruzl [day] - rez /rez/ [vine]

1.3. The Phonetic process of vowels

One characteristic of Bayzai dialect which distinguishes it from Standard Persian is using final vowel "a" instead of the final vowel "e" in Standard Persian

Example:

Bayzai

āmoxta

rafta

xuna

\section{Standard Persian}

āmuxte learned

rafte gone

xune house 
TABLE 1:

OTHER VOWEL CHANGES OF BAYZAI DIALECT IN COMPARISON WITH THE STANDARD FARSI ARE

\begin{tabular}{|c|c|c|c|c|}
\hline Standard Persian & & Bayzāyi & & \\
\hline [e] & & [i] & & \\
\hline šeš & $/ \mathrm{se} /$ & šiš & $/ \int \overline{\mathrm{i}} \mathrm{j} /$ & Six \\
\hline kelid & /kelīd/ & kilil & $/ \mathrm{kin} \overline{1} \mathbf{1} /$ & key \\
\hline konjed & /k ndzed/ & konjit & /kondzīt/ & sesame \\
\hline [e] & & [o] & & \\
\hline gereftan & /gereftæn/ & geroftan & /geroftæn/ & to take \\
\hline šemordan & /femordæn/ & šomordan & /fomordæn/ & to count \\
\hline emru & /emru:/ & omru & /omru:/ & to count \\
\hline emsāl & /ems $\Lambda 1 /$ & omsāl & /omsal/ & this year \\
\hline [a] & & {$[\overline{\mathrm{a}}]$} & & \\
\hline arus & /ærus/ & āris & /arīs/ & \\
\hline ammā & /æm / & $\bar{a} \mathrm{a} \bar{a}$ & /ama/ & but \\
\hline$\overline{\mathrm{amu}}$ & /æmu:/ & $\overline{\mathrm{a} m u}$ & /amu/ & uncle \\
\hline [a] & & {$[\mathrm{o}]$} & & \\
\hline aždahā & /æ3dæha/ & oždahā & /ozdæha/ & \\
\hline abrišam & /æbrīyæm/ & abrišom & /æbrījom/ & silk \\
\hline kadu & /kædu:/ & kodi & /kədī/ & \\
\hline$[\bar{a}]$ & & [o] & & \\
\hline āyene & /ajənə/ & oyna & /ojna/ & mirror \\
\hline pāyiz & /pajīz/ & poyiz & /pojīz/ & autumn \\
\hline tapāle & /tæpalə/ & tapola & /tæpolə/ & dung \\
\hline$[\overline{\mathrm{a}}]$ & & [e] & & \\
\hline barādar & /bær $\Lambda$ dær/ & beredar & /beredær/ & brother \\
\hline sāye & $/ \mathrm{s} \Lambda \mathrm{j} / /$ & seya & /sejæ/ & shadow \\
\hline$[\mathrm{u}]$ & & [i] & & \\
\hline balut & /bælut/ & balit & /bælīt/ & oak \\
\hline xun & /khun/ & xin & /khīn/ & blood \\
\hline $\mathrm{mu}$ & /mu:/ & $\mathrm{mi}$ & $/ \mathrm{mi}: /$ & hair \\
\hline
\end{tabular}

\subsection{Consonants}

By using the substitution method, the consonants of the dialect can be categorized as:

2.1.1./p/ : pulmonic, outward, tense, voiceless, aspirated, plosive, oral, bilabial.

1./p/-/b/ : pa:r /par/ [to weed out] - ba:r /bar/ [draw]

2./p/-/m/ : kap /kæp/ [novel] - kam /kæm/ [few (little)]

3./p/-/t/ : par /pær/ [feather] - tar /tær/ [wet]

2.1.2./b/ : pulmonic, outward, lax, voiced, plosive, oral, bilabial.

$1 . / \mathrm{b} /-/ \mathrm{p} /:$ bus /bus/ [kiss] - pus /pus/ [skin]

2./b/-/m/ : bāl /bal/ [wealth] - māl /mal/ [property]

3./b/-/d/: [pretext] - [seed]

2.1.3./f/ : pulmonic, outward, tense, voiceless, fricative, oral, labio- dental

1./f///v/: safā /sæfa/ [serenity] - savā /sæva/ [morning]

2./f/-/s/: $\mathrm{kaf} / \mathrm{kæf} /$ [floor] - kas /kæs/ [person]

3./f///čl : fāl /fal/ [omen] - čāl /t fall/ [nest]

2.1.4. /v/: pulmonic, outward, lax, voiced, fricative, oral, labio-dental

1./v///b/: vaxt/væght/ [time] - baxt /bækht/ [fortune]

2./v/-/r/: vā /va/ [open] - rā /ra/ [day]

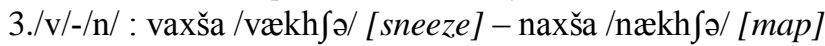

2.1.5./t/ : pulmonic - outward, tense, voiceless, aspirated, passive, oral, alveo - palatal

1. /t///p/ : tenja /tendzə/ [sprout] - penja /pendzə/ [finger]

2. /t/-/d/ : tar /tær/ [wet] - dar /dær/ [door]

3. $/ \mathrm{t} / / / \mathrm{k} /: \mathrm{kol} / \mathrm{kJl} /[$ slow] - tol $/ \mathrm{tol} /[$ hill]

2.1.6. /d/ : pulmonic, outward, voiced, plosive, oral, alveo - dental

1. /d/-/t/: dir /dīr/ [far] - tir /tīr/ [arrow]

2. /d/-/n/: dār/dar/ [tree] - nār/nar/ [pomegranate]

3. /d/-/g/: del /del/ [heart] - gel /gel/ [flower]

2.1.7. /s/ : pulmonic, outward, tense, voiceless, fricative, oral, alveo-dentel

1./s/-/z/ : sir /sìr/ [garlic (satisfied)] - zir /zīr/ [under]

2./s/-/d/ : sar/sær/ [head] - dar/dær/ [door]

3./s/-/š/ : si /si:/ [for] - ši /fi:/ [slope] 
2.1.8. /z/ : pulmonic, outward, tense, voiced, fricative, oral, alveolar

1. /z/-/s/ : za:rā /zæ?ra/ [zahra] - sa:rā /sæ?ra/ [desert]

2. /z/-/1/ : gāz /gaz/ [a kind of nail] - gāl /gal/ [loose]

3. /z/-/r/ : gāz /gaz/ [a kind of nail] - gār /gar/ [end]

2.1.9. $/ \check{\mathrm{s}} /$ : pulmonic, outward, tense, voiceless, oral, alveo - palatal

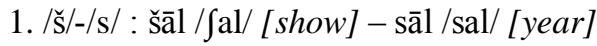

2. $/ \check{\mathrm{s}} /-/ \mathrm{x} /$ : šow / Jou/ [night] - xow /khou/ [sleep]

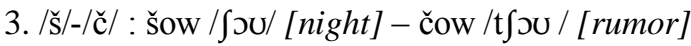

2.1.10. /ž/ : pulmonic, outward, lax, voiced, fricative, oral, alveo - palatal $^{1}$

1. /ž/-/r/ : možda /məzdə/ [glad tidings] - morda /mordə/ [dead]

2.1.11./y/ : semi-vowel, pulmonic, outward, lax, voiced, approximant, oral, velar

1. /y/-/d/ : ya /jæ/ [one] - da /dæ/ [other]

2. /y/-/p/ : yala /jælə/ [oblique] - pala /pælə/ [foolish]

3. /y/-/t/ : yorya /jorghə/ [trot] - torya /torghə/ [a kind of bird]

2.1.12. /č/ : pulmonic, outward, tense, voiceless, fricative, plosive, oral alveo - palatal

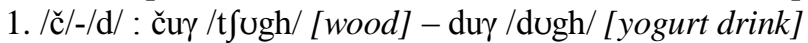

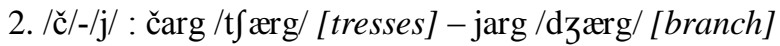

3. /č/-/k/ : čika /t fīkə/ [leakage] - tika /tîkə/ [piece]

2.1.13. /j/ : pulmonic, outward, lax, voiced, fricative, plosive, alveo- palatal

1. $/ \mathrm{j} /$-/g/ : jerow /dzerou/ [socks] - gerow /gerou/ [bet]

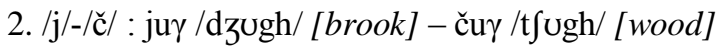

3. /j/-/r/ : jud /dzud/ [year] - rud /rud/ [good child]

2.1.14. / $/$ / : Pulmonic, outward, tense, voiceless, aspirated, plosive, oral, palatal

1. /k/-/p/ : kuza /kuzə/ [jar] - puza /puzə/ [cape (prow)]

2. $/ \mathrm{k} /-/ \mathrm{d} /: \mathrm{kār} / \mathrm{kar} /$ [work] - tār /tar/ [dark (cord)]

3. /k/-/g/: kend /kend/ [hip] - gend /gend/ [knot]

2.1.15. /g/ : pulmonic, outward, plosive, oral, palatal

1. /g/-/k/ : gerd /gerd/ [round] - kerd /kerd/ [the root of the past - tense verb "go"]

2. /g/-/d/ : gard /gærd/ [dust] - dard /dærd/ [ache]

3. /g/-/q/ : gabr /gæbr/ [guebre ( zoroastrian)] - qabr /ghæbr/ [grave]

2.1.16. $/ \mathrm{x} /$ : pulmonic, outward, tense, voiceless, fricative, oral, palatal

1. /x/-/k/ : xār /khar/ [thorn] - kār /kar/ [work]

2. /x/-/h/ : xiz /khīz/ [jump] - hiz /hīz/ [lecherous]

3. /x/-/q/ : xows /khous/ [sleep] - qows /ghous/ [arc]

2.1.17. /?/ : pulmonic, outward, tense, voiceless, plosive, oral, glottal ${ }^{2}$

1. /?/-/b/ : abr /æbr/ [cloud] - babr /bæbr/ [tiger]

2. /?/-/k/ : orsi /orsi:/ [shoes] - korsi /korsi:/ [chair]

3. /?/-/m/ : ašk /æ $\int \mathrm{k} /$ [tear] - mašk /mæ $\int \mathrm{k} /$ [leather bottle]

2.1.18. $/ \mathrm{m} /$ : pulmonic, outward, lax, voiced, bilabial, nasal

$1 . / \mathrm{m} /-/ \mathrm{b} /: \mathrm{moz} / \mathrm{moz} /$ [wage] - boz /boz/ [goat]

2. $/ \mathrm{m} /-/ \mathrm{p} /:$ menj /mendz/ [fog] - penj /pendz/ [toe]

3. $/ \mathrm{m} /-/ \mathrm{n} /$ : jum /dzum/ [clothes] - jun /dzun/ [soul]

2.1.19./n/ : pulmonic, outward, lax, voiced, alveo-dental, nasal

1. /n/-/t/ : nar /nær/ [male] - tar /tær/ [wet]

2. $/ \mathrm{n} /-/ \mathrm{b} /$ : nana /nænə/ [mother] - bana /bænə/ [mountain almond]

3. /n/-/d/ : nun /nun/ [bread] - dun /dun / [ ......]

2.1.20. /1/ : pulmonic, outward, lax, voiced, lateral, alveolar

1. /1/-/r/: gel /gel/ [cloy] - ger /ger/ [pretext]

2. /1/-/k/ : leštan /le $\int \mathrm{tæn} /$ [to put] - keštan /kejtæn/ [to plant]

3. /l/-/q/ : lem /lem/ [made] - qem /ghem/ [funnel]

2.1.21. /h/ : pulmonic, outward, tense, voiceless, fricative, oral, glottal

1. In Bayzai dialect, the existence of consonant (t) cannot be proved by using the minimal pairs, but there are some original words in which there exists the consonant/ž/, like: oždahā [dragon], hažda [eighteen].

2. $\gamma$ and $\mathrm{q}$ are the different allophones of one phoneme. When they come before or after a consonant, they are considered as phoneme. When /?/ comes at the beginning of a word, its presence or absence makes no signifact change and it is just an arbitrary (Najafi, Abu-al-Hasan, 1995 , pp. 69 78) 
1. /h/-/x/ : hār /har/ [rabid] - xār /khar/ [thorn]

2. /h/-/q/ : holla /hollə/ [club] - qolla /ghollə/ [crest of mountain]

3. /h/-/d/ : havā /hæva/ [air, sky] - davā /dæva/ [drug]

2.1.22. /q/ : pulmonic, outward, lax, voiced, plosive, oral, palatal

1. /q/-/k/ : qulak /ghulæk/ [small ghoul (giant)] - kulak /kulæk/ [shoulder]

2. /q/-/h/ : qiz /ghīz/ [jump] - hiz /hīz/ [lecherous]

3. /q/-/g/ : qowda /ghoudə/ [bunch] - gowda /goudə/ [hollow]

2.1.23. /r/ : pulmonic, outward, lax, voiced, trill, oral, alveolar

1. /r/-/l/ : rās /ras/ [right] - lās /las/ [dog]

2. $/ \mathrm{r} /-/ \mathrm{m} /: \mathrm{rā} / \mathrm{ra} /$ [way] - mā /ma/ [moon]

3. $/ \mathrm{r} /-/ \mathrm{z} /$ : bor /bor/ [group] - boz /boz/ [goat]

TABLE 2:

CONSONANTS OF BAYZAI DIALECT

\begin{tabular}{|c|c|c|c|c|c|c|c|c|}
\hline Manner of articulation & bilabial & labio-dental & dental & alveolar & alveo-palatal & velar & uvular & glottal \\
\hline Plosive (stop) & $\mathrm{p}, \mathrm{b}$ & & $t, d$ & & & $\mathrm{k}, \mathrm{g}$ & $q$ & $\delta$ \\
\hline Fricative & & $f, v$ & & $\mathrm{~s}, \mathrm{z}$ & žšs, & & $\mathrm{x}$ & $\mathrm{h}$ \\
\hline Fricative-plosive & & & & & $\mathrm{j}$ č, & & & \\
\hline Trill & & & & $\mathrm{r}$ & & & & \\
\hline Nasal & $\mathrm{m}$ & & & $\mathrm{n}$ & & & & \\
\hline Approximant & & & & $\mathrm{L}$ & & $\mathrm{y}$ & & \\
\hline
\end{tabular}

2.2. phonetic process of consonants

In Bayzai dialect, as opposed to Standard Persian, some conversions have occurred. 
TABLE 3:

SOME EXAMPLES OF PHONETIC PROCESS OF CONSONANTS ARE:

\begin{tabular}{|c|c|c|c|c|c|c|c|}
\hline & & Bayzai & & & Standard Persian & & \\
\hline 1 & $d<t:$ & došak & $/ \mathrm{d} \mathrm{\int} æ \mathrm{k} /$ & $<$ & tošak & /tofæk/ & Mattress \\
\hline 2 & $\mathrm{t}<\mathrm{d}:$ & maččet & 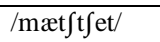 & $<$ & masjed & /mæsdzed/ & mosque \\
\hline 3 & ss $<$ st : & ossā & /ossa/ & $<$ & ostād & /ostad/ & master \\
\hline 4 & $\mathrm{x}<\gamma:$ & ossoyun & /ossoghun/ & $<$ & ostoxān & /ostıkhan/ & bone \\
\hline 5 & $x<q:$ & farx & /færkh/ & $<$ & farq & /færgh/ & difference \\
\hline 6 & $q<x:$ & qiz & /ghīz/ & $<$ & xiz & /khīz/ & jump \\
\hline 7 & $\gamma<\mathrm{g}:$ & teyerg & /tegherg/ & $<$ & tagarg & /tægærg/ & hail \\
\hline 8 & $y<g:$ & diya & $/$ dīæ/ & $<$ & digar & /dīgær/ & other \\
\hline 9 & $f<b:$ & $\tan \overline{\mathrm{f}} \mathrm{f}$ & /tænaf/ & $<$ & tanāb & /tænab/ & rope \\
\hline 10 & $\mathrm{~m}<\mathrm{b}:$ & kesm & /kesm/ & $<$ & kasb & $/ \mathrm{kæsb} /$ & acquiring \\
\hline 11 & $\mathrm{v}<\mathrm{b}:$ & dovāla & /dovala/ & $<$ & dobāre & /dobara/ & again \\
\hline 12 & $\mathrm{~b}<\mathrm{v}:$ & dib & $/ \mathrm{d} \overline{\mathrm{i}} \mathrm{b} /$ & $<$ & div & $/ \mathrm{d} \overline{\mathrm{i}} \mathrm{v} /$ & beast \\
\hline 13 & $\mathrm{~m}<\mathrm{n}:$ & ambār & /æmbar/ & $<$ & anbār & /ænbar/ & storehouse \\
\hline 14 & $\mathrm{~L}<\mathrm{n}:$ & Lifa & $/ / \bar{i} f \mathfrak{x} /$ & $<$ & nife & $/ \operatorname{nīfə/} /$ & roof \\
\hline 15 & $\mathrm{n}<\mathrm{m}:$ & bun & /bun/ & $<$ & bām & $/ \mathrm{bam} /$ & chicory \\
\hline 16 & $\check{\mathrm{s}}<\mathrm{s}:$ & kāšni & /kafnī/ & $<$ & kāsni & /kasnī/ & chicory \\
\hline 17 & $\check{\mathrm{s}}<\mathrm{j}:$ & moštabā & $/ \mathrm{m} \int \mathrm{ftæba} /$ & $<$ & mojtabā & /modftæba/ & proper name \\
\hline 18 & $1<r:$ & zanjil & /zændzīl// & $<$ & zanjir & /zændzīr/ & chain \\
\hline 19 & $\mathrm{r}<1:$ & šekāl & /Sekal/ & $<$ & šekār & /Sekar/ & hunt \\
\hline 20 & $\mathrm{r}<1:$ & taraktol & /tæræktol/ & $<$ & terāktor & /teraktor/ & tractor \\
\hline 21 & $\mathrm{x}<\mathrm{h}:$ & bextar & /bekhtær/ & $<$ & behtar & /behtær/ & better \\
\hline 22 & $\mathrm{~b}<\mathrm{y}:$ & gerba & /gerbə/ & $<$ & gerya & /gerja/ & cry \\
\hline 23 & $\mathrm{r}<\mathrm{y}:$ & šur & //ur/ & $<$ & šuy & $/ \int u j /$ & wash \\
\hline 24 & $\mathrm{~d}<\mathrm{y}:$ & hamsāda & /hæmsadə/ & $<$ & hamsāya & /hæmsaja/ & neighbor \\
\hline 25 & $x<y:$ & morx & /morkh/ & $<$ & mory & /morgh/ & hen \\
\hline 26 & $\mathrm{q}<\gamma:$ & qorbat & /ghorbæt/ & $<$ & 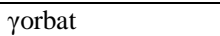 & /ghərbæt/ & life in exile \\
\hline 27 & $1<d:$ & kilil & /kîlilil/ & $<$ & kelid & /kelīd/ & key \\
\hline 28 & $\mathrm{~d}<\mathrm{t}:$ & yord & /jord/ & $<$ & yurt & /jurt/ & camp \\
\hline 29 & $\mathrm{t}<\mathrm{d}:$ & konjit & /kכndzīt/ & $<$ & konjed & /kondzed/ & sesame \\
\hline 30 & ss $<$ st : & bassa & /bæsə/ & $<$ & baste & /bæstə/ & close \\
\hline 31 & ss $<$ st : & dassak & /dæsæk/ & $<$ & dastak & /dæstæk/ & handle \\
\hline 32 & $x<q:$ & boxča & /bokhtfa/ & $<$ & bpqče & /boght $\int \partial /$ & bundle \\
\hline 33 & $q<x:$ & masqara & /mæsghæræ/ & $<$ & masxare & /maskhære/ & ridiculous \\
\hline 34 & $\gamma<\mathrm{g}:$ & Layat & /læghæt/ & $<$ & Lagad & /lægæd/ & kick \\
\hline 35 & $\mathrm{y}<\mathrm{g}:$ & aya & /æjə/ & $<$ & agar & /ægær/ & if \\
\hline 36 & $\mathrm{f}<\mathrm{b}:$ & jārruf & /dzaruf/ & $<$ & jārub & /dzarub/ & broom \\
\hline 37 & $\mathrm{~m}<\mathrm{b}:$ & kamutar & /kæmutær/ & $<$ & kabutar & /kæbutær/ & pigeon \\
\hline 38 & $\mathrm{v}<\mathrm{b}:$ & ševet & // evet/ & $<$ & šebet & //ebet/ & \\
\hline 39 & $\mathrm{~b}<\mathrm{v}:$ & hombār & /hombar/ & $<$ & hamvār & /hæmvar/ & flat \\
\hline 40 & $\mathrm{~m}<\mathrm{n}:$ & ambor & /æmbər/ & $<$ & anbor & /ænbər/ & a pair of tongs \\
\hline 41 & $\mathrm{~L}<\mathrm{n}:$ & Lilufar & /līlufær/ & $<$ & nilufar & /nīlufær/ & morning glory \\
\hline 42 & $\check{\mathrm{s}}<\mathrm{s}:$ & šātir & //atīr// & $<$ & sātur & /satur/ & chopper \\
\hline 43 & $\check{\mathrm{s}}<\mathrm{j}:$ & paštā & /pæfta/ & $<$ & panjtā & /pændzta/ & five \\
\hline 44 & $1<\mathrm{r}:$ & balg & /bælg/ & $<$ & barg & /bærg/ & leaf \\
\hline
\end{tabular}

In Bayzai dialect, in respect of syllable structure of the words, some phonetic changes could be observed in some separate cases. This change is: the conversion or omission of one consonant in the paired consonants in the middle and at the end of the words:

1. consonant " $t$ " in group " $\mathrm{s}$ " $\rightarrow$

$\begin{array}{lll}\text { xassa /khæsə/ } & <\text { xaste /khæstə/ } & \text { tired } \\ \text { ossā /ossa/ } & <\text { ostād /ostad/ } & \text { master } \\ \text { dāsun /dasun/ } & <\text { dāstān/dastan/ } & \text { story } \\ \text { rās /ras/ } & <\text { rāst /rast/ } & \text { right } \\ \text { 2. consonant "d" in group "rd, zd, nd": } & \\ \text { ārbiz /arbīz/ } & <\text { ārdbiz /ardbīz/ } & \text { sieve } \\ \text { bolan /bolæn/ } & <\text { boland /bolænd/ } & \text { high } \\ \text { moz /moz/ } & <\text { mozd /mozd/ } & \text { wage } \\ \text { ruxuna /rukhunə/ } & <\text { rudxune /rudkhunə/ } & \text { river }\end{array}$

3. consonant "m, n" in group "sm, ns":
čiš
$<$ češm
< čašm 


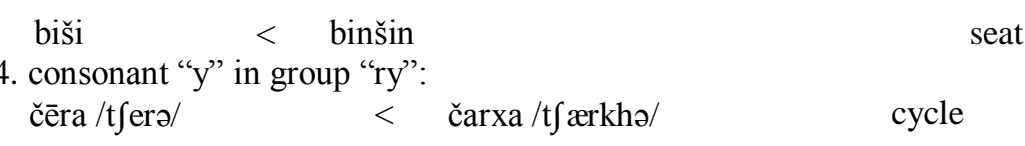

The omission of the consonants after the short and long vowels:

$\begin{array}{llll}\text { omru } & >\text { emruz } & \text { today } & \text { eye } \\ \text { a } & <\text { az } & \text { from } & \text { seat } \\ \text { zā } & >\text { vāz } & \text { bāz } & \text { open } \\ \text { engā /enga/ } & <\text { engār /engar/ } & & \text { as } \\ \text { doxta /dəkhtə/ } & <\text { doxtar/dəkhtær/ } & & \text { girl } \\ \text { ya /jə/ } & >\text { yak/jæk/ } & & \text { one }\end{array}$

In Bayzai dialect, the consonant " $h$ " is added before the vowel at the beginning of some words:

\begin{tabular}{|c|c|c|c|}
\hline hešār /he Jar/ & $<$ & ešāra /e Jarə/ & \\
\hline hassom /hæsəm/ & $<$ & assom /æsım/ & skimmer \\
\hline hafi /hæfi/ & $<$ & af'i /æfí/ & viper \\
\hline helāj /heladz/ & $<$ & elāj /eladz/ & cure \\
\hline \multicolumn{4}{|c|}{ ometimes " $h$ " is omitted from the end of the words: } \\
\hline $\mathrm{mā} / \mathrm{ma} /$ & $<$ & $\mathrm{māh} / \mathrm{mah} /$ & moon \\
\hline $\mathrm{pi} / \mathrm{p} \overline{\mathrm{i}} /$ & $>$ & pih /pīh/ & tallow \\
\hline $\mathrm{ko} / \mathrm{kJ} /$ & $>$ & kuh /kuh/ & mountain \\
\hline čā /t $\int \mathrm{a} /$ & $<$ & čāh /t $\int a h /$ & well \\
\hline $\mathrm{ru} / \mathrm{ru} /$ & $<$ & ruh /ruh/ & spirit \\
\hline jāgā /dzaga/ & $>$ & jāygāh /dzajgah/ & position \\
\hline
\end{tabular}

The " $h$ " consonant is also omitted from the middle of some words and because of which the word has compensatory extension.

$\begin{array}{llll}\text { ko:na /kənə/ } & > & \text { kohne /kohnə/ } & \text { old } \\ \text { ba:ra /bæ?ra/ } & > & \text { bahre /bæhrə/ } & \text { profit } \\ \text { da:na /dænə/ } & > & \text { dahane } & \text { opening } \\ \text { sa:rā/særa/ } & > & \text { sahrā /sæhra/ } & \text { desert } \\ \text { za:rā /zæ?ra/ } & > & \text { zahrā /zæhra/ } & \text { Proper name }\end{array}$

The omission of the consonant " $h$ " from the middle and the end of the Arabic loanwords occurs according to the phonetic rules of Bayzai dialect example:

\begin{tabular}{|c|c|c|c|}
\hline ma:mud /mæ?mud/ & $>$ & mahmud /mæhmud/ & (Proper name) \\
\hline mobā /moba/ & $>$ & mobāh /mobah/ & permissible \\
\hline ta:vil & $>$ & tahvil & delivery \\
\hline $\begin{array}{l}\text { e:yānan /e?janæn/ } \\
\text { towfa /t’ufə/ }\end{array}$ & $\begin{array}{l}> \\
<\end{array}$ & $\begin{array}{l}\text { ehyānan /ehjanæn/ } \\
\text { tohfe /tohfə/ }\end{array}$ & $\begin{array}{l}\text { by any chance } \\
\text { gift }\end{array}$ \\
\hline mo:kam /mı?kæm/ & $>$ & mohkam /mohkæm/ & firm \\
\hline ma:kum /mæ?kum/ & $>$ & mahkum /mæhkum/ & Convicted criminal \\
\hline na:r /næ?r/ & $>$ & nahr /næhr/ & stream \\
\hline
\end{tabular}

a) Consonant distribution

All the consonants of Bayzai dialect can occur at the beginning, in the middle or at the end of a syllable, except " $h$ " which can be omitted from the end of the syllable, e.g. "feqh" (Islamic jurisprudence) which is pronounced "feq" and "čāh" (well)- čā, "rāh" (way, road) - rā , "māh" (moon) - mā , "ruh" (soul, spirit) - ru.

b) Vowel distribution:

All the vowels of Bayzai dialect can occur at the beginning, in the middle or at the end of a syllable.

1-3-1- Phonetactics and Syllable construction

In Bayzai dialect, like standard Farsi, no word has consonant cluster. In the beginning position many words of this dialect have 2-consonant cluster in the beginning or middle position, but 3- consonant clusters are not common in this dialect. For example, the word "tambar" [postage stamp] is pronounced so in Persian, but "tambar" in Bayzai dialect

Different kinds of syllables in this dialect are: cv, cvc, cvec , like: pā (foot), pir (old), pors (ask).

\section{B. Word Formation (Morphology)}

2.1. Male and female: In this dialect, there is no gender distinction for nouns. But according to the farm husbandry nature of the region, there are different names for different kinds of male and female animals. For example for different kinds of "cow", the following words there exit.

Male $\rightarrow$ jonga /dəzngə/, varzow /værzou/, toxmi /tokhmī/ 


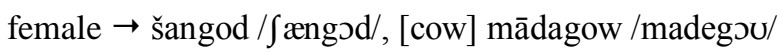

2.2. singular and plural

The plural is constructed by adding the suffixes "un" and "-hā" in which "h" is omitted: men, women, trees

2.3. Definite and indefinite

The definite suffixes are "-u" and "-a", like:

Bilâ (that shovel), the same as Burjani, Māsarmi and Samghani dialects.

And "-u" like: doxtaru /dəkhtæru/ (that girl), like most of the dialects of Fars province as well as the Shiraz dialect.

The indefinite mark is "-i" (with or whitout "one")

Morqi /morghī/ (a hen), ya deraxti /derakhtī/ (one tree)

Sometimes phonetic change is accompanied by adding a go-between consonant:

Ya mardey /mærdej/ (a man ), ya bačey /bæt fej/ (a child)

2.4. Pronouns

2.4.1. Disjunct personal pronouns are:

$\mathrm{mo} / \mathrm{ms} /(\mathrm{ma})\{\mathrm{I}, \mathrm{we}\}$, to $/ \mathrm{t} \mathrm{s} /(\mathrm{you}), \mathrm{u} / \mathrm{u} /$ (he/she),

$\mathrm{mā} / \mathrm{ma} /$ (we), šomā / Joma/ (you), unā /una/ (they), inā /īna/ (they)

2.4.2. Conjunct personal pronouns are:

-om /om/ (my), et /et/ (your), eš /e J/ (aš) \{his/her\}

-emun /emun/ (our), -etun /etun/ (your), -ešun /efun/ (their)

2.4.3. Demonstration pronouns:

$\mathrm{i} / \overline{1} /$ (this), u /u/ (that), hami /hæmī/ (this one), hamu /hæmu/ (that one).

2.4.4. Interrogation pronouns:

ki /kī/ (who), key /kej/ (when), siče /sīte/ (why)

2.4.5. Indefinite pronouns:

Diya /dìjə/ (other), hama /hæmæ/ (all)

2.5. Number

Generally, the numeral system of this dialect is the same as Farsi but some interesting cases are:

Doyyom /dəjjom/ (second), seyyom /sejjom/ (third)

Just in these 2 cases, "yyom" is used. In other cases, only "om" is used:

2.6. verb

2.6.1. In using simple past, present prefect, past continuous, past perfect, subjunctive past (past subjunctive ), present indicative, present subjunctive, and imperative, apart from phonetic changes. Bayzai dialect is the same as Standard Persian. Conjugation of a verb is based on 2 verb substances: past substance and present substance.

Examples for the simple past are: singular: raf, raft, raftam (he/she/I/you went)

Plural: raftand, raftin, raftim, (they/you/we went)

Examples for the present indicative: singular: mire, mirey, miram (he she is going, you're going, I'm going), plural: miran, mirin, mirim (they/you/we are going).

The verb "must" in singular third person in the auxiliary verbs is used a "ba": mibā rafta bāše $\rightarrow$ s/he must have gone.

The verb "Dadan" (give) is conjugated without "h" in present tense: singular: mide, midim, midam (s/he gives, you / I give)

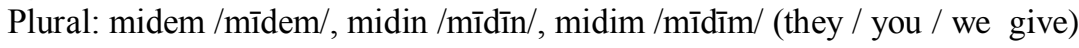

2.6.2. verb prefixes

There exit 2 types of verb prefixes:

1. continuous prefixes "mi" (both for present continuous and past continuous):

migi /mīgī/ (you say): migofti /mīgoftī/ (you were saying)

2. full prefix (for subjunctive and imperative) which is in from of be, bo, bu, bi:

bezan /bezæn/ (hit), bokoš /bokəf/ (kill), budun /budun/ (know), biyow /bījou/ (come).

2.6.3. verb suffixes:

The suffixes of present indicative and present subjunctive are:

2.6.3.1. Indicative

Singular: 1.am (om) 2. i/ey 3. a(e) plural: 1. im (eym) 2. in (eyn) 3. en (an)

2.6.3.2. In singular second person of past intransitive verbs, "t" disappears

Singular: raftom /ræftom/ (I went), rafti /ræftī/ (you went), raf/ræf/ (he/she went)

Plural: poxtom /pokhtom/ (I cooked), poxti /pokhtī/ (you cooked), pox /pokh/ (s/he cooked)

An example of conjugating verb is:

Singular: kerdom /kerdom/ (I did), kerdi /kerdī/ (you did), ker /ker/ (s/he did)

Plural: kardim /kærdīm/ (we did), kardin /kærdīn/ (you did), karden /kærden/ (they did)

The verb "hasten" (being) in singular third person is in the from of "he" 
Singular: hesom /hesom/ (I am), hesi /hesī/ (you are), he (s) /hes/ (s/he is)

Plural: hesim /hesīm/ (we are), hesin /hesīn/ (you are), hesen /hesen/ (they are)

And its negative from is:

Singular: nisom /n̄̄som/ (I'm not), nisi /n̄̄sī/ (you're not), ni /nī/ (s/he is not)

Plural: nisim /nīsīm/ (we're not), nisin /n̄̄sīn/ (you're not), nisen /nīsen/ (they’re not)

2.7. derivation:

2.7.1. The most important derivative suffixes are:

- $\mathrm{i} \rightarrow$ Make adjective from mass noun e.g.

howlaki /adj, houlækī/ (hastily), bixod /adj, bīkhod/ (of poor quality),

- ak which is a relative pronoun suffix meaning similarity:

āssak /asæk/ (mill), toxmak/tokhmæk/ (roasted seed)

-aki: which is relative suffix $\rightarrow$ maidenhood, bovine (pertaining to cow)

-uk: which is relative adj $\rightarrow$ the male of a plant, ivy

-ka which makes noun: tofka /tıfkæ/ (spit), bālaka /b $\Lambda$ lækæ/ (niche)

2.8. syntax

Generally, the syntax of Bayzai is the same as Farsi's.

The Bayzai vocabularies are divided into 3 groups:

3.1. The Arabic and Turkish loanwords which come to this dialect through the standard language:

" talab [claim], sarf /særf/ [consuming], ra:m /ræm/ [composition], qassam/ghæsæm/ [oath]" are same examples of Arabic loanwords and words "il [a nomadic tribe], ordu /ordu/ [camp], qešlāq /ghe llagh/ [winter quarter], yeylāq /jejlagh/ [summer quarter], qoroy /ghorogh/ [forhidden]" are some Turkish loanwords.

3.2. There are some words which are common in both Bayzai dialect and standard Farsi:

šow / fou/ [night], ruz/ruz/ [day], āsemun /asemun/ [sky], bača /bætfə/ [child], koh /koh/ [mountain], del/del/ [heart]

3.3. words which are exclusive to this dialect, or at least are not used in standard Farsi anymore and which even do not exist in the comprehensive encyclopedias such as Dehxoda Encyclopedia. Therefore, these words can be listed as the exclusive vocabularies of this dialect. The number of these vocabularies is very large, so just some examples are given here:

ārg /arg/ [Palate], axiya /ækhīja/ [rumination], bārizi /barīzī/ [a gift from bridegroom to the bride], bošma /bofmə/ [hedge], bogol /bogol/ [a cylindrical container to keep flour and salt], pāxtan /pakhtæn/ [cutting back branches], tabru /tæbru/ [re-growing of the plants], bāhenda /bahendə/ [bird], xāg /khag/ [egg], rašt /ræft/ [ash], regā /rega/ [separate], sener /sener/ [it's a kind clay to build a wall], kotorom /kotวrom/ [sick], koč /kəft/ [chin], korpa /kərpa/ [not grown], kener /kener/ [pick], kijik /kīdz̄īk/ [button], got/got/ [great], gaštan/gæ/tæn/ [to bite], gener/gener/ [knot], gowān /gəvan/ [a kind of skirt], hilu /hīlu:/ [endurance (swing)], yakābas /jækabæs/ [excellent], digna /dīgnə/ [yesterday], doxtan /dokhtæn/ [to milk], tandār /tænd $\Lambda \mathrm{r} /$

\section{DISCUSSION}

There is no considerable difference between Bayza dialect and standard Persian. The observable quantitative differences are naturally the continuation of phonetic evolution of Bayza dialect. There is no qualitatively marked difference between the phonologic and phonetic systems of Bayza and standard Persian. For example:

$\mathrm{Y} \rightarrow \mathrm{g}$ or $\mathrm{X} \rightarrow \mathrm{q}$ or $\mathrm{ss} \rightarrow \mathrm{st}$ or $\mathrm{d} \rightarrow \mathrm{t}$

The evolutions are not that effective to lead speakers of two dialects to have a difficalty interacting. The description and analysis of Bayza dialect can be effective and helpful due to the recognition of some unknown aspects of Persian. Fot example, in terms of investigating the phonetic evolution of $r \rightarrow y$ and also comparing the Bayza word شور، شستن 'shostan', 'shoor' respectively with the word 'شوي' 'shooy' in standard Persian, we historically come to this point that the root of this word has involved the final consonant and the same origin of its Avestan equivalent 'šu $\theta$ ra:

Examining the phonetic evolution of $\mathrm{m} \rightarrow \mathrm{n}$ and comparing the word ambar in Bayza dialect with the word anbar in Persian showed that the consonant $/ \mathrm{n} /$ in standard Pertion is the next evolution of the prefix 'ham' in Old Persian and the consonant $/ \mathrm{m} /$ still exists in Bayza dialect.

Nevertheless, some of the words that still exist in Bayza dialect are nowadays dead in standard Persian and can help us identify some of the verbs and nouns in standard Persian. Verbs like 'باختن' 'Bakhtan' or nouns like tandār, sener, gowan do not exist in any published dictionary or glossary.

\section{CONCLUSION}

1. Bayzai dialect, is a branch of southwest Iranian dialects.

2. In this dialect there exist 3 short vowels, 3 long vowels and 4 diphthongs.

3. There is no difference in the pronunciation of the short and long vowels in Bayzai dialect and standard Farsi. 
4. Bayzai tense or lax consonant makes no meaning distinction in this dialect.

5. Hamze/?/ has just a distinctive role and its presence or absence makes no meaning distinction.

6. Always voiceless consonants are in contrast with the voiced consonants.

7. In this dialect, no syllable begins with a consonant cluste.

8. In terms of structure, Bayzai dialect is similar to Farsi, but it preserves its phonetic dependence and differences.

9. Plural morphemes in this dialect are ān and hā, in which $\mathrm{h}$ is sometimes omitted.

10. Ezafe suffix (mark), like Farsi, is e

11. Indefinite suffixes are "ey" and "i" which come after noun and "u" is the definite suffix.

12. The object identifiers are "na" or "a" which come after noun

13. There is no grammatical gender in this dialect.

14. Bayzai vocabularie's have been disappeared (died) by passing the time and given their place to the new ones.

15. In using Bayzai vocabulary and idioms, many words have been preserved in the tradition and costume of the people life.

16. Many of these vocabs are not used in standard Farsi any more, so they can be regarded as a great treasure for future studies.

\section{REFERENCES}

[1] Bailey, H. and B. (2002). Nesā and fasā: Trans. Fateme Jadali, Sorush, Pirmoghan, Jamshid Sorushyar's memorial (collection of articles) by Katāyun Mazdāpour. Tehran: Sorayyā.

[2] Eshmit, R.(2004). A guide to Iranian Farsi languages, 2 vols, Trans. Hasan Rezaei, Bāghbidi , et al. Tehran: Ghoghnus.

[3] Frye, R. (1989). Ancient history of Iran: Tehran: Elmi-o-Farhangi Publication.

[4] Hovar, K. (1996). Iran and Persian culture: Trans. Hasan Anushe. Tehran: Amirkabir.

[5] Ibn-e-Hughal, A. M. (1987). "Surt-at-Arz", (Ibn-e-Hughal's travelogue). Trans. Jafar Sheār. Tehran: Amirkabir.

[6] Ibrahimi, Gh. (2004). The culture of Bayza people. Tehran: Nik kherad.

[7] Ibrahimi, Gh. (2004). Anzan, Anshan. Persian studies of Kerman, shahid Bahonar University.

[8] Najafi, A. (1995). Basics of linguistics and its application in Persian language. Tehran: Niloufar.170.

[9] Oranski, V. (1999). "Persian languages". trans. Ali Ashraf Sadeqi . Tehran: Sokhan.

[10] Samare, Y. (1989). Phonetics in Persian language. Tehran: Nashre Daneshgahi.

[11] Tabari, M.I.J. (1983). Tarikhe Tabari: trans. Abolghasem Payand.Tehran: Asatir.

Ghorbanali Ebrahimi is a lecturer at Islamic Azad University, Najafabad Branch. He has been teaching various Persian Literature courses at different Universities. His areas of interest are: Persian Literature, Persian poetry, and Linguistics. 